\title{
Impending scope of Water Sanitation and Hygiene (WASH) in the post COVID19 pandemic era: An opportunity call
}

\section{Pachillu Kalpana ${ }^{1}$, Poonam Trivedi ${ }^{2}$, Krupali Patel ${ }^{3}$, Sandul Yasobant ${ }^{4}$, Deepak Saxena ${ }^{5}$}

${ }^{1}$ Lab-In-Charge, IIPHG-NIDHI-TBI, Indian Institute of Public Health Gandhinagar (IIPHG), Gandhinagar, Gujarat, India; ${ }^{2} J u n i o r$ Epidemiologist, IIPHG-NIDHI-TBI, Indian Institute of Public Health Gandhinagar (IIPHG), Gandhinagar, Gujarat, India; ${ }^{3}$ Junior Researcher, One Health Graduate School, Center For Development Research (ZEF), Bonn, Germany; ${ }^{4}$ Junior Researcher, One Health Graduate School, Center For Development Research (ZEF), Bonn, Germany; ${ }^{5}$ Professor, Indian Institute of Public Health Gandhinagar (IIPHG), Gandhinagar, Gujarat, India

Abstract $\underline{\text { Introduction }} \underline{\text { Methodology }} \underline{\text { Results }} \underline{\text { Conclusion }}$
Corresponding Author
Prof. (Dr.) Deepak Saxena, Indian Institute of Public Health Gandhinagar (IIPHG), Opposite Air
Force Headquarters, Near Lekawada Bus Stop, Gandhinagar-Chiloda Road, Lekawada, P.O. CRPF
Camp- 382042, Gandhinagar, Gujarat, India
E Mail ID: ddeepak72@iiphg.org

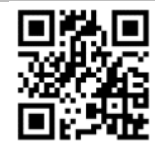

\section{Citation}

Kalpana P, Trivedi P, Patel K, Yasobant S, Saxena D. Impending scope of Water Sanitation and Hygiene (WASH) in the post COVID19 pandemic era: An opportunity call. Indian J Comm Health. 2020;32(2-Special Issue):244247.

Source of Funding: Nil Conflict of Interest: None declared

\section{Article Cycle}

Received: 12/04/2020; Revision: 15/04/2020; Accepted: 16/04/2020; Published: 20/04/2020 This work is licensed under a Creative Commons Attribution 4.0 International License.

\section{Abstract}

COVID19 pandemic is now testing health systems of countries globally irrespective of geographical location, environmental conditions, and population demographics. Among various prevention strategies as suggested by WHO and others, hand hygiene has emerged as the most important public health preventive measure to control similar outbreaks in the past (SARS \& MERS) also including the current COVID19 pandemic. The COVID19 pandemic provides a well-timed opportunity to stress the importance of water, sanitation, and hygiene (WASH) in the healthcare facilities (HCFs) and optimize infection prevention control across the globe. It also gives an opportunity to extend beyond conventional WASH and includes domains of biomedical waste management, Infection Prevention, and Control activities and also environmental hygiene. This perspective piece elaborates on the importance of optimal WASH and future scopes in HCFs during the post COVID19 pandemic era.

\section{Keywords}

WASH; COVID-19; Pandemic; LMIC

\section{The past pandemics: Lesson learned}

The emergence of the initial cases of severe pneumonia in Wuhan, China found out to be the novel coronavirus infection, which rapidly blown out to the national and international boundaries of the world causing an outpouring of the COVID-19(1). On 30th January 2020, the novel coronavirus Severe Acute Respiratory Syndrome-Coronavirus-2 (SARSCoV-2) was declared as the sixth public health emergency of international concern(2); followed a pandemic by the Director-General of the World
Health Organization (WHO) on 11th March 2020(3). Evidence indicates a weaker pathogenic picture of i.e. SARS-CoV2, than previous coronavirus SARS but a stronger transmission competence(4).Epidemiological investigation of 2003 SARS outbreak revealed that the transmission between 128 cases linked to healthcare-associated infections(5). In 2012, the Middle East Respiratory Syndrome (MERS) is another coronavirus that also caused the outbreak within the hospital reported from in several countries. Coronavirus can be 
transmitted by direct contact between the infected and susceptible host whereas the environment also plays a significant role in cross contamination(7). Research also indicated that fomite-mediated transmission to have a potential significance in respiratory viral disease(8). Study also shows that even a single occurrence of hand contact with contaminated surface can transfer pathogen easily and cause recontamination. Hence the low compliance to the hand hygiene of health care workers (HCWs) is a big worry(9). These pathogens can survive on healthcare facility (HCF) surfaces but the destructibility due to proper cleaning process has evidence (10). The regular microbiological screening of HCFs is what it needs along with proper hand hygiene and environmental cleaning. Previous pandemics came up with the importance of infection, prevention and control (IPC) measures and training of HCWs as a cost-effective way to prevent the spread of MERS-CoV in HCFs(6). Evidence also stresses on the importance of the optimal WASH practices HCFs to prevent the pandemic outbreaks $(11,12)$. Washing hands with soaps are the most effective way and are proven to be significant in reducing the residual viruses or bacteria on hands $(13,14)$.

\section{The current COVID19 pandemic: An opportunity}

COVID-19 pandemic outbreak threatening millions of lives is transmitted through indiscernible respiratory droplets coming out into the air from cough or sneeze of an infected person(15). These droplets in the air can be inherited to close by individuals or it can drop on inanimate surfaces(4). Research shows the novel coronavirus can persist on inanimate surfaces like metals, glass or plastic for up to 9 days(16). These invisible viruses present on inanimate surfaces can infect others when they touch their eyes, nose or mouth. The SARS-CoV-2 infection is described with an incubation period of between 1-14 days (4). Public health interventions are playing an important role, synchronized with time and active actions will aid in averting further cases or poor health consequences. One of the way to early prevention could be to reduce the transmission risk by recognition of cases early(17). The epidemiological studies shows that contact transmission is one of the main routes of the SARSCov-2 and hand hygiene is considered the most important public health preventive measure(14). The WHO has also emphasized the importance of hand hygiene as a critical component of health reliability $(18,19)$. Evidence highlights the important response to various pandemic outbreak requires hand hygiene compliance by everyone $(1,20)$.

Investigating opportunities: Ways to prevent future pandemics

One of the most cost-effective strategies for increasing pandemic preparedness, especially in resource-constrained settings, is investing in core public health infrastructure, including water and sanitation systems. Better Water, Sanitation \& Hygiene (WASH) and waste management practices, if consistently applied can serve as barriers to human-to-human transmission of the COVID-19 virus in homes, communities, health care facilities, schools, and other public spaces(21). WASH includes the basics of infection prevention measures and emphasizes environmental hygiene and personal hygiene including hand washing being the most important ones. The persistence of microorganisms and recent coronaviruses on hard surfaces and viable transmission via these is been a proven point now. This concludes the importance of environmental hygiene in HCFs in reducing healthcare-associated infections. The importance of proper disinfection of HCF instruments is extremely important not only during the epidemic period but also afterward to reduce the risk of cross infections(22). The perception of clean or not clean surfaces depends on individuals whereas what if the clean surface is also not microbiologically clean(23). The recontamination of the hands after cleaning is an understanding depending on the assumption about various disinfecting procedures including the type of surface, frequency, method, and training of the neglected cleaning staff (24).WASH practices are important during pandemic outbreaks like this novel coronavirus but are always a priority for improving quality care, strengthening the health systems and ensuring the safety of patients as well and the important healthcare workers. Recent data shows that in Low and middle-income countries (LMIC), $50 \%$ of HCFslack piped water, $33 \%$ lack improved sanitation, 39\% lack adequate infectious waste disposal and $73 \%$ lack sterilization equipment(25). As per the global baseline report 2019, one in four healthcare facilities lacks basic water services and one in five has no sanitation services impacting 2.0 billion and 1.5 billion people, respectively(26). WHO report suggested that although awareness of the importance of hand hygiene in preventing infection with the COVID-19 virus is high, access to hand 
hygiene facilities that include alcohol-based hand rubs as well as soap and water is often suboptimal in the community and HCF settings, especially in LMICs(27). Further, global data from 48 countries shows that only $60 \%$ of HCFs reach the criteria of segregating waste(26), which emphasize the importance of BioMedical Waste (BMW) as part of WASH strategy during post-pandemic time and reflecting urgent need for strengthening and monitoring WASH in HCFs. The importance of addressing inadequacies in facility based WASH and IPC is becoming more acute given the outbreak of infectious disease like COVID 19.

\section{WASH as a prime opportunity: Improving in HCFs}

WASH practices are one of the prime approaches to improve the healthcare quality in HCFs. Although, there are several tools for assessment of WASH in HCFs are available, our review indicates that none of the WASH tools are comprehensive and integrated for the assessment (28). In this situation, the WASH ToolBox plus(29), which has been developed and validated for rapid assessment of healthcare settings in India might provide a golden opportunity to scale up the assessment. WASH that addresses beyond the water quality together with sanitation, hygiene, biomedical waste management, and environmental hygiene needs to be promoted. This tool can easily be used for assessment of WASH at facility levels or department levels. There is a prerequisite for an additional exertion in ensuring WASH in HCFs. Developing mental models for training, monitoring and giving essential feedbacks on clear guidance for understanding and differentiating between clean and not clean surfaces would be more effective. The most prominently including all the staff of the HCFs healthcare facilities from officers to the cleaners is utmost. The cleaners are always a neglected workforce playing a major role in infection, prevention and control practices(30). The hand hygiene protocols should be followed by all individuals involved in patient care by the aid of change agents in the HCFs such as managers and colleagues. One of the methods known as "knock" is also suggested by research to be time-efficient and replicable for hand hygiene purposes[19]. A behavioral change among the healthcare workers and a user- friendly environment that can facilitate hand hygiene should be promoted. There can be more chances of adding to these points for mainly the sustainability reason for WASH in HCFs but not the less. During the epidemics strengthening the healthcare settings is the most important task and robust research can help and guide public health policies. The use of technologies wisely and costeffective innovations for resource-limited HCFs would also play an important role in sustainable WASH in HCFs.

\section{The way forward: Optimal WASH into HCFs}

Getting optimal WASH into HCFs is an urgent global health need, which needs to be strengthened at the local, regional and global levels. WASH should not be looked at as a component of the HCFs, it should be integrated with the HCF activities and should be promoted. It includes the commitment to the ongoing training, handholding to each cadre of the healthcare workers, and microbiologically healthy environment. We urge to promote a WASH userfriendly environment that facilitates hygiene and reduces opportunities for recontamination in the HCFs.

\section{Authors Contribution}

All authors have contributed equally.

\section{References}

1. Ma Q-X, Shan H, Zhang H-L, et al. Potential utilities of mask wearing and instant hand hygiene for fighting SARS-CoV-2. Journal of Medical Virology

2. Eurosurveillance Editorial Team. Note from the editors: World Health Organization declares novel coronavirus (2019-nCoV) sixth public health emergency of international concern. Euro surveillance: bulletin Europeen sur les maladies transmissibles = European communicable disease bulletin 2020;25. doi:10.2807/1560-7917.ES.2020.25.5.200131e

3. The Lancet. Palliative care and the COVID-19 pandemic. The Lancet. 2020;395:1168. doi:10.1016/S01406736(20)30822-9

4. Tian HY. 2019-nCoV: new challenges from coronavirus. Zhonghua Yu Fang Yi Xue Za Zhi 2020;:E001-E001.

5. Varia M, Wilson S, Sarwal $S$, et al. Investigation of a nosocomial outbreak of severe acute respiratory syndrome (SARS) in Toronto, Canada. CMAJ 2003;169:285-92.

6. Chowell G, Abdirizak F, Lee S, et al. Transmission characteristics of MERS and SARS in the healthcare setting: A comparative study. BMC Medicine 2015;13:210. doi:10.1186/s12916-015-0450-0

7. Kraay ANM, Hayashi MAL, Hernandez-Ceron N, et al. Fomite-mediated transmission as a sufficient pathway: A comparative analysis across three viral pathogens 11 Medical and Health Sciences 1117 Public Health and Health Services. BMC Infectious Diseases 2018;18. doi:10.1186/s12879-018-3425-x

8. Boone SA, Gerba CP. Significance of fomites in the spread of respiratory and enteric viral disease. Applied and Environmental Microbiology. 2007;73:1687-96. doi:10.1128/AEM.02051-06 
9. Fomites and Infection Transmission / Infection Control Today.

10. Dancer SJ. The role of environmental cleaning in the control of hospital-acquired infection. Journal of Hospital Infection. 2009;73:378-85. doi:10.1016/j.jhin.2009.03.030

11. Weber N, Martinsen AL, Sani A, et al. Strengthening Healthcare Facilities Through Water, Sanitation, and Hygiene (WASH) Improvements: A Pilot Evaluation of wASH FIT in Togo. Health Security 2018;16:S24-45. doi:10.1089/hs.2018.0042

12. Huttinger A, Dreibelbis R, Kayigamba F, et al. Water, sanitation and hygiene infrastructure and quality in rural healthcare facilities in Rwanda. BMC Health Services Research 2017;17:517. doi:10.1186/s12913-017-2460-4

13. Breidablik HJ, Lysebo DE, Johannesse L, et al. Effects of hand disinfection with alcohol hand rub, ozonized water or soap water - time for reconsideration? Journal of Hospital Infection

14. Ran L, Chen X, Wang Y, et al. Risk Factors of Healthcare Workers with Corona Virus Disease 2019: A Retrospective Cohort Study in a Designated Hospital of Wuhan in China. Clinical infectious diseases : an official publication of the Infectious Diseases Society of America

15. World Health Organization. Coronavirus disease (COVID-19): Situation Reports. WO Director-General's Special envoys on COVID-19 preparedness and response. 2020;:111.https://www.who.int/emergencies/diseases/novelcoronavirus-2019/situation-reports (accessed $11 \mathrm{Apr}$ 2020).

16. Kampf G, Todt D, Pfaender S, et al. Persistence of coronaviruses on inanimate surfaces and its inactivation with biocidal agents. Journal of Hospital Infection 2020.

17. Patel A, Jernigan DB, nCo VCDCRT. Initial Public Health Response and Interim Clinical Guidance for the 2019 Novel Coronavirus Outbreak - United States, December 31, 2019-February 4, 2020. MMWR Morb Mortal Wkly Rep 2020;:140-6.

18. Organization $\mathrm{WH}$. WHO guidelines on hand hygiene in health care: first global patient safety challenge: clean care is safer care. 2009.

19. Rush JL, Flowers RH, Casamiquela KM, et al. The knock: An adjunct to education for improving outpatient hand hygiene. Journal of the American Academy of Dermatology 2016;75:231-3. doi:10.1016/j.jaad.2016.01.027

20. Wong VWY, Cowling BJ, Aiello AE. Hand hygiene and risk of influenza virus infections in the community: $A$ systematic review and meta-analysis. Epidemiology and Infection. 2014;142:922-32. doi:10.1017/S095026881400003X

21. World Bank . WASH (Water, Sanitation \& Hygiene) and COVID-19. WASH interventions for effective COVID19 pandemic response. 2020;101:1.https://www.worldbank.org/en/topic/wate r/brief/wash-water-sanitation-hygiene-and-covid-19 (accessed 11 Apr 2020).
22. Zhang $M C$, Xie $H T$, $X u K K$, et al. Suggestions for disinfection of ophthalmic examination equipment and protection of ophthalmologist against 2019 novel coronavirus infection. Zhonghua Yan Ke Za Zhi 2020;:E001-E001.

23. Trivedi $P$, Patel $K$, Yasobant $S$, et al. Visually clean is not necessarily microbiologically safe: Learnings from WASH assessment of maternity units of Gujarat, India. Journal of Family Medicine and Primary Care 2020;9:788. doi:10.4103/jfmpc.jfmpc_1066_19

24. Gon G, Dancer S, Dreibelbis R, et al. Article Type: Letter to the Editor Reducing hand recontamination of health workers during COVID-19. doi:10.1017/ice.2020.111

25. USAID. Water, Sanitation and Hygiene (WASH), Maternal Child Survival Program. 2019.

26. World Health Organization and The United Nations Children's Fund. WASH in health care facilities: Global baseline report 2019. Geneva: 2019. doi:CC BY-NC-SA 3.0 IGO

27. Mk W, Fronti S, Haemorrhagic F, et al. Recommendation to Member States to improve hand hygiene practices widely to help prevent the transmission of the COVID-19 virus by : 1 . Providing universal access to public hand hygiene stations and making their use obligatory on entering and leaving any. 2020;:2-4.

28. Patel K, Kalpana P, Trivedi P, et al. Assessment of water, sanitation and hygiene in HCFs: Which tool to follow? Reviews on Environmental Health. 2019. doi:10.1515/reveh-2019-0001

29. Patel K, Yasobant S, Trivedi P, et al. Water Sanitation \& Hygiene (WASH) Tool Box plus for rapid assessment of Healthcare facilities: Experiences from India. Gandhinagar: 2020. dx.doi.org/10.17504/protocols.io.bceaitae

30. Cross S, Gon G, Morrison E, et al. An invisible workforce: the neglected role of cleaners in patient safety on maternity units. Global Health Action 2019;12. doi:10.1080/16549716.2018.1480085 